\title{
Comparison of Eplerenone and Spironolactone in Treatment of Patients with Congestive Heart Failure Visiting a Tertiary Hospital in North India
}

\author{
Abhishek Walia', Anil Kem², Jaison Paul Sharma ${ }^{3}$ \\ 1,2,3Saraswati Institute of Medical Sciences, Hapur (U.P.), India. \\ DOI: https://doi.org/10.24321/2349.7181.201811
}

\begin{abstract}
Background: Congestive heart failure (CHF) is a serious clinical condition, with not many established
treatment options. Eplerenone (Inspra) is the second aldosterone antagonist commonly used (in preference
to Spironolactone) for treatment of hypertension and CHF in the US. Literature for its effectiveness in India
is lacking, hence the need for the present study.
Methodology: The present cross-sectional, observational study was done among 100 adult patients
(>20 years) (50 patients in each study group: Eplerenone and Spironolactone), diagnosed with chronic
CHF and poor left ventricle function (ejection fraction <40\%) unknown of their blood pressure status.
Sociodemographic profile, clinical and laboratory status was evaluated which included blood pressure
measurements, lipid profile, kidney function tests, and other vitals. Assessment of parameters was done
after 8 weeks of treatment.
Results: Systolic blood pressure, diastolic blood pressure and CK levels showed a significant change over
the study duration, with Eplerenone producing a better result than Spironolactone (p<0.05). Remaining
parameters showed a non-significant difference.
Conclusion: Eplerenone group proved to be associated with a better outcome in hypertension and
cardiovascular parameters; however, due to limitations in sample selection, more research is warranted.
Keywords: Eplerenon, Spironolactone, Congestive heart failure, Hypertension
\end{abstract}
\section{Introduction}

Congestive heart failure (CHF) is a clinical condition characterized by lack of perfuse systematic circulation due to the heart's damaged pumping capabilities. ${ }^{1,2} \mathrm{CHF}$ affects nearly 5 million people each year, in which at least 500,000 are newly diagnosed cases. ${ }^{3} \mathrm{CHF}$ is traditionally viewed as a chronic condition that is the result of silent killers such as hypertension and unmanaged diabetes. However, more recently, clinical research has focused on CHF cases that occur suddenly. ${ }^{1} \mathrm{CHF}$ has always been a keen area of research due to its high rate of mortality and morbidity as well as being the leading cause of hospitalization in the elderly. ${ }^{4}$

Unfortunately, most forms of heart failure (those due to damaged heart muscle) have no known cure. The treatments available aim to improve the patient's quality of

Corresponding Author: Dr. Abhishek Walia, Saraswati Institute of Medical Sciences, Hapur (U.P.), India.

E-mail Id: karralsaabmbbs@gmail.com

Orcid Id: https://orcid.org/0000-0002-6925-9518

How to cite this article: Walia A, Kem A, Sharma JP. Comparison of Eplerenone and Spironolactone in Treatment of Patients with Congestive Heart Failure Visiting a Tertiary Hospital in North India. J Adv Res Med 2018; 5(2): 29-35. 
life as well as length of survival. ${ }^{1}$ These treatments comprise drug therapy and, more importantly, lifestyle changes by the patient. The recommended changes consist of: quitting smoking (if applicable), losing weight (if necessary), abstaining from alcoholic beverages, reducing salt and fat intake, and regular physical activity.

In addition to this, a doctor through follow-up appointments must closely monitor the patient. Furthermore, a majority of heart failure patients have to take medications to help the heart in its ability to pump. The common medications are ACE (angiotensin converting enzyme) inhibitors, diuretics, digitalis glycosides, aldosterone antagonists, angiotensin receptor blockers (ARBs), and beta-blockers. Some patients who suffer from the more severe forms of $\mathrm{CHF}$ require the implantation of devices such as IABPs (intra-aortic balloon pumps) or LV ADs (left ventricular assist devices) to assist with their management.

Eplerenone (Inspra) is the second aldosterone antagonist commonly available in the US. It received Food and Drug Administration (FDA) approval in 2002 and 2003, for the treatment of hypertension and heart failure, respectively. Eplerenon blocks the mineral corticoid receptors in the kidney, heart, blood vessels and brain more selectively than Spironolactone (another aldosterone antagonist; it has progestational and anti-androgenic adverse effects due to its nonspecific binding to various steroid receptors. ${ }^{5}$

Adverse reactions of Eplerenone are gastrointestinal intolerance, dizziness, hypercalemia and hypertriglyceridemia. ${ }^{6}$ Breast discomfort and enlargement or other anti-androgen effects are seen in men, treated with Spironolactone but are infrequent with Eplerenon. ${ }^{7}$ Although its use is common in the US and other developed parts of the world, its effectiveness in Indian scenario is debatable. Hence, the intention of this study is to study the effect of Eplerenon (Inspra): second Aldosterone antagonist in patients of CHF visiting a tertiary care hospital in North India.

\section{Methodology}

The present cross-sectional observational study was done in a tertiary care hospital in North India from July 2015 to June 2017 among 100 adult patients (>20 years of age), diagnosed with chronic CHF unknown of their blood pressure status. Selected patients essentially had poor left ventricle function (ejection fraction $<40 \%$ ) of any cause. Patients with congenital heart malformations, other chronic comorbidity such as uncontrolled diabetes mellitus, patients with life expectancy less than 1 year, patients who had a myocardial infarction less than 4 weeks prior to enrollment in the study, overt renal disease, cancer or suspicion of malignancy, pregnant patients and patients with hypertensive emergency were excluded.
Ethical approval was taken from the Institutional Ethical Committee after explaining the aims and objectives of the study. A written informed consent was obtained from each patient for the study. The involvement of the subjects was voluntary and deliberate.

All patients underwent a clinical and laboratory evaluation. The demographic data were obtained from a questionnaire survey. Following variables were assessed: gender, age (years), ethnicity, occupation, education, income, socioeconomic status (calculated with combination of occupation, income and education in accordance with modified Kuppuswamy scale, 2016)..$^{8}$ Other parameters evaluated were: body mass index (BMI), pulse, blood pressure (BP), weight and temperature in Fahrenheit scale. Data on comorbidities such as dyslipidemia, cancer, and diabetes were also noted.

Blood samples were obtained during the time of patient reporting for serum electrolytes. Estimation of hemoglobin by hematocrit, serum creatinine, BUN, uric acid, RBS, enzymes and serum lipid profile was also done by the principle of colorimetry using spectrophotometer.

\section{Study Groups}

Patients already undergoing treatment from physicians in the hospital were equally randomized in two treatment groups: Eplerenone group and Spironolactone group of 50 subjects each. Patient compliance was noted and patients were asked to keep a record of medications taken. Biochemical and BP evaluation was performed at follow-up visits and compared at the end of 8-week period. Loss to follow-up and non-compliance was insignificant.

\section{Study Factors}

- Vitals of the individual: pulse, weight, temperature

- Lipid profile: total cholesterol, LDL and triglyceride levels

- Serum creatinine and blood urea nitrogen (BUN) levels

- Serum electrolytes ( $\mathrm{Na}, \mathrm{K}$ )

- $\quad$ Systolic and diastolic blood pressure

- ECG and echocardiogram findings; noted as normal/ abnormal

\section{Statistical Analysis}

Statistical analysis was performed using SPSS v17.0. Differences between parametric groups were compared by the Student-t test; correlation was done by Pearson's correlational analysis. Non-parametric variables were evaluated using chi-square test for statistical comparisons.

\section{Results}

Majority of study participants were in the 31-50 year age group, with a mean of $51.3 \pm 9.7$ years and range $44-72$ 
years. Majority were males (87\%) and $13 \%$ females. Most of the study participants were married (89\%), $9 \%$ were unmarried and $2 \%$ divorced/separated. More than three-fourths of subjects (79\%) belonged to middle class, followed by $14 \%$ in lower-middle class, $5 \%$ in upper-middle and $2 \%$ in upper class (Table 1 ).

Table 1.Sociodemographic Characteristics

\begin{tabular}{|c|c|c|c|c|}
\hline \multicolumn{2}{|c|}{ Sociodemographic Characteristics } & Frequency & Percent (\%) & \multirow{5}{*}{$\begin{array}{c}\mathbf{p} \text {-value } \\
0.127\end{array}$} \\
\hline \multirow[t]{4}{*}{ Age group (years) } & $31-40$ & 8 & 8 & \\
\hline & $41-50$ & 26 & 26 & \\
\hline & $51-60$ & 45 & 45 & \\
\hline & $>60$ & 21 & 21 & \\
\hline \multirow[t]{2}{*}{ Gender } & Males & 87 & 87.0 & \multirow[t]{2}{*}{0.884} \\
\hline & Females & 13 & 13.0 & \\
\hline \multirow[t]{3}{*}{ Marital status } & Married & 89 & 89 & \multirow[t]{3}{*}{0.291} \\
\hline & Unmarried & 9 & 9 & \\
\hline & Divorced/Separated & 2 & 2 & \\
\hline \multirow[t]{4}{*}{ Socio-economic status } & Lower-middle & 14 & 14 & \multirow[t]{4}{*}{0.929} \\
\hline & Middle & 79 & 79 & \\
\hline & Upper-middle & 5 & 5 & \\
\hline & Upper & 2 & 2 & \\
\hline
\end{tabular}

The mean pulse rate of study participants was $77.2 \pm 8.74$ (range 64-94). The mean weight of study participants was $88.9 \pm 10.2 \mathrm{~kg}$ (range $75-111 \mathrm{~kg}$ ). Mean body temperature of study participants was $97.8 \pm 0.9$ (range 96-100). Systolic BP had a mean value of $132.8 \pm 9.4$; mean diastolic BP was $92.4 \pm 4.3$. Tables 2 and 3 show descriptive statistics of baseline levels of study parameters.

Table 2.Descriptive Statistics of Study Parameters

\begin{tabular}{|c|c|c|c|c|c|c|}
\hline & \multicolumn{2}{|c|}{ Blood Pressure } & \multicolumn{2}{|c|}{ KFT } & \multirow[t]{2}{*}{ CK } \\
\hline & & Systolic & Diastolic & Blood Urea & Serum Creat & \\
\hline \multicolumn{2}{|l|}{$\mathrm{N}$} & 100 & 100 & 100 & 100 & 100 \\
\hline \multicolumn{2}{|c|}{ Mean } & 132.8 & 92.43 & 45.33 & 2.283 & 195.74 \\
\hline \multicolumn{2}{|c|}{ SE } & .0572 & .1695 & 1.236 & .0548 & 2.163 \\
\hline \multicolumn{2}{|c|}{ Median } & 133 & 94 & 43.50 & 2.300 & 192.00 \\
\hline \multicolumn{2}{|c|}{ SD } & 9.43 & 4.35 & 13.537 & .6002 & 23.697 \\
\hline \multicolumn{2}{|c|}{ Minimum } & 122 & 74 & 25 & 1.0 & 159 \\
\hline \multicolumn{2}{|c|}{ Maximum } & 156 & 104 & 81 & 3.2 & 241 \\
\hline \multirow[t]{3}{*}{ Percentiles } & 25 & 132 & 80 & 32.00 & 1.900 & 182.00 \\
\hline & 50 & 138 & 88 & 43.50 & 2.300 & 192.00 \\
\hline & 75 & 152 & 102 & 51.00 & 2.700 & 211.00 \\
\hline
\end{tabular}

Table 3.Descriptive Statistics of Study Parameters

\begin{tabular}{|c|c|c|c|c|c|c|c|c|c|}
\hline & Total Cholesterol & HDL & LDL & Triglyceride & SGOT & SGPT & LDH & Alk Phosp \\
\hline \multicolumn{2}{|l|}{$\mathrm{N}$} & 120 & 120 & 120 & 120 & 120 & 120 & 120 & 119 \\
\hline \multicolumn{2}{|l|}{ Mean } & 246.08 & 41.19 & 138.69 & 199.38 & 35.918 & 57.74 & 242.31 & 126.00 \\
\hline \multicolumn{2}{|l|}{$\mathrm{SE}$} & 3.582 & .290 & 2.779 & 2.595 & .6178 & 1.059 & 2.979 & 1.872 \\
\hline \multicolumn{2}{|c|}{ Median } & 251.00 & 41.00 & 130.00 & 200.00 & 38.300 & 61.00 & 231.00 & 128.00 \\
\hline \multicolumn{2}{|c|}{ SD } & 39.242 & 3.176 & 30.437 & 28.423 & 6.7677 & 11.605 & 32.629 & 20.426 \\
\hline \multicolumn{2}{|c|}{ Minimum } & 185 & 37 & 95 & 159 & 22.7 & 34 & 185 & 95 \\
\hline \multicolumn{2}{|c|}{ Maximum } & 308 & 47 & 195 & 249 & 47.0 & 73 & 293 & 166 \\
\hline \multirow[t]{3}{*}{ Percentiles } & 25 & 208.00 & 39.00 & 108.00 & 171.00 & 29.100 & 50.00 & 217.00 & 106.00 \\
\hline & 50 & 251.00 & 41.00 & 130.00 & 200.00 & 38.300 & 61.00 & 231.00 & 128.00 \\
\hline & 75 & 280.00 & 44.00 & 169.50 & 223.00 & 40.100 & 69.00 & 275.00 & 139.00 \\
\hline
\end{tabular}


Table 4 shows the baseline statistics among study participants in terms of ECG, Echo findings and serum electrolytes in both the treatment groups using unpaired-t test. At the baseline, the mean abnormal ECG was $57 \%$ and $54 \%$ in Eplerenone and Spironolactone group, respectively. Similarly, 62\% of the subjects had ventricular hypertrophy in Eplerenone group and 59\% in Spironolactone group. Regurgitation was seen in 28\% subjects in Eplerenone group and $30 \%$ in Spironolactone group. The mean baseline ejection fraction was 36\% (2.2 SD) in Eplerenone group and $34 \%$ (4.1 SD) in Spironolactone group (Fig. 1). None of the baseline characteristics was statistically different among study groups.

Table 4.Estimates of Baseline Study Parameters among Two Treatment Groups

\begin{tabular}{|c|c|c|c|c|c|c|c|c|c|c|c|c|}
\hline \multirow{2}{*}{ Baseline } & \multicolumn{2}{|c|}{ ECG } & \multicolumn{2}{c|}{ Ejec Frac } & \multicolumn{2}{c|}{$\begin{array}{c}\text { Ventricular } \\
\text { Hypertrophy }\end{array}$} & \multicolumn{2}{c|}{ Regurgitation } & \multicolumn{2}{c|}{ Serum Na } & \multicolumn{2}{c|}{ Serum K } \\
\cline { 2 - 14 } & Normal & Abnormal & Mean & SD & Yes & No & Yes & No & Mean & SD & Mean & SD \\
\hline Eplerenone & $43 \%$ & $57 \%$ & $36 \%$ & 2.2 & $62 \%$ & $38 \%$ & $28 \%$ & $72 \%$ & 137.2 & 4.1 & 4.8 & 0.5 \\
\hline $\begin{array}{c}\text { Spironolac- } \\
\text { tone }\end{array}$ & $46 \%$ & $54 \%$ & $34 \%$ & 4.1 & $59 \%$ & $41 \%$ & $30 \%$ & $70 \%$ & 139.1 & 5.8 & 5.1 & 0.8 \\
\hline p-value & \multicolumn{2}{|c|}{0.463} & \multicolumn{2}{|c|}{0.984} & \multicolumn{2}{|c|}{0.743} & \multicolumn{2}{c|}{0.672} & 0.712 & 0.135 \\
\hline
\end{tabular}

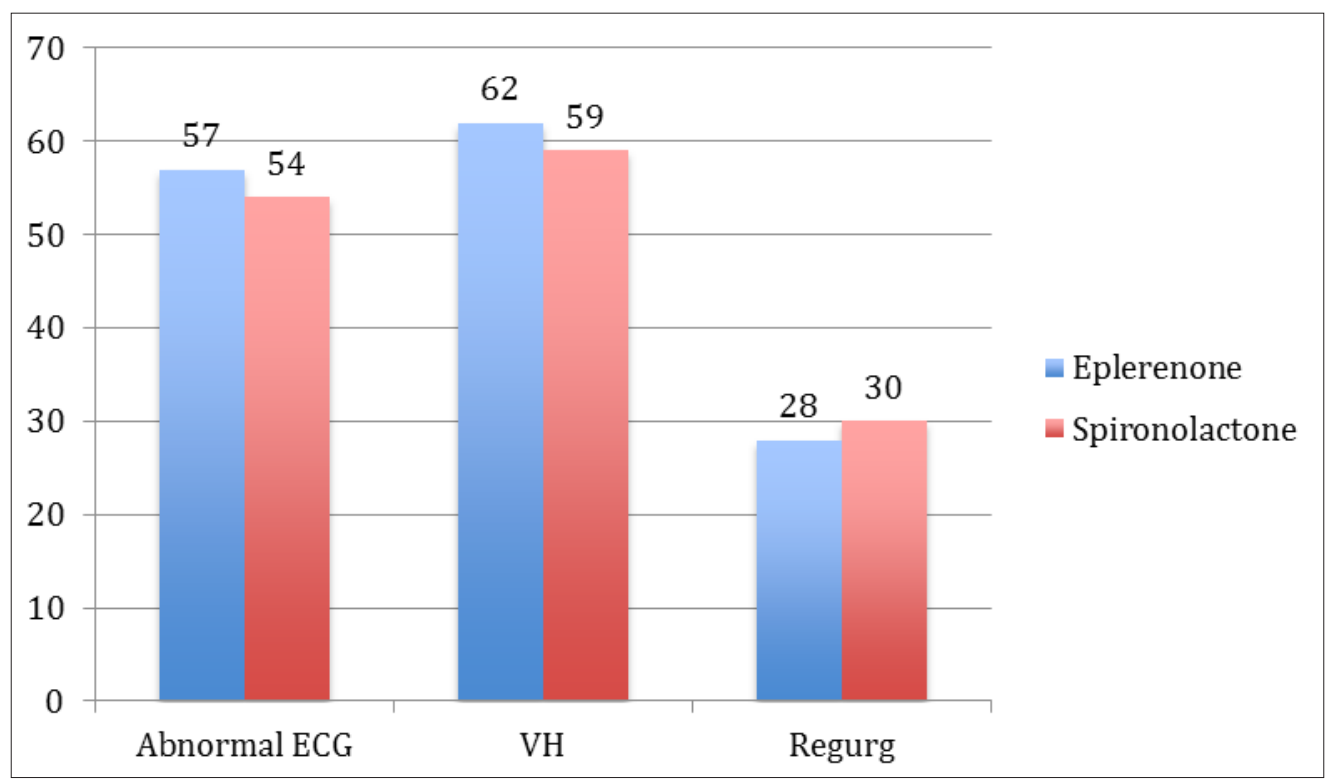

Figure 1.Baseline Study Parameters among Sample Population

Table 5 shows the 8-week estimates among study participants in terms of ECG, Echo findings and serum electrolytes in both the treatment groups using unpaired-t test. The mean abnormal ECG prevalence was 51\% in Eplerenone group and $49 \%$ in Spironolactone group. Nearly $41 \%$ of subjects had ventricular hypertrophy in Eplerenone group and $45 \%$ in Spironolactone group.
Both these parameters were significantly different among study groups. Regurgitation was seen in $22 \%$ of subjects in Eplerenone group and 28\% in Spironolactone group, not statistically significant. The mean baseline ejection fraction was 54\% (3.1 SD) in Eplerenone group and 52\% (2.7 SD) in Spironolactone group, statistically significant (Fig. 2).

Table 5.Estimates of 8-week Study Parameters among Two Treatment Groups

\begin{tabular}{|c|c|c|c|c|c|c|c|c|c|c|c|c|}
\hline \multirow[t]{2}{*}{ Baseline } & \multicolumn{2}{|c|}{ ECG } & \multicolumn{2}{|c|}{ Ejec Frac } & \multicolumn{2}{|c|}{$\begin{array}{l}\text { Ventricular } \\
\text { Hypertrophy }\end{array}$} & \multicolumn{2}{|c|}{ Regurgitation } & \multicolumn{2}{|c|}{ Serum $\mathrm{Na}$} & \multicolumn{2}{|c|}{ Serum K } \\
\hline & Normal & Abnormal & Mean & SD & Yes & No & Yes & No & Mean & SD & Mean & SD \\
\hline Eplerenone & $49 \%$ & $51 \%$ & $54 \%$ & 3.1 & $41 \%$ & $59 \%$ & $22 \%$ & $78 \%$ & 155.7 & 3.7 & 3.5 & 0.3 \\
\hline $\begin{array}{l}\text { Spironolac- } \\
\text { tone }\end{array}$ & $51 \%$ & $49 \%$ & $52 \%$ & 2.7 & $45 \%$ & $55 \%$ & $28 \%$ & $72 \%$ & 152.3 & 4.1 & 4.1 & 0.5 \\
\hline$p$-value & \multicolumn{2}{|c|}{$0.003^{*}$} & \multicolumn{2}{|c|}{$0.041^{*}$} & \multicolumn{2}{|c|}{ 0.039* } & \multicolumn{2}{|c|}{0.371} & \multicolumn{2}{|c|}{$0.002^{*}$} & \multicolumn{2}{|c|}{$0.015^{*}$} \\
\hline
\end{tabular}




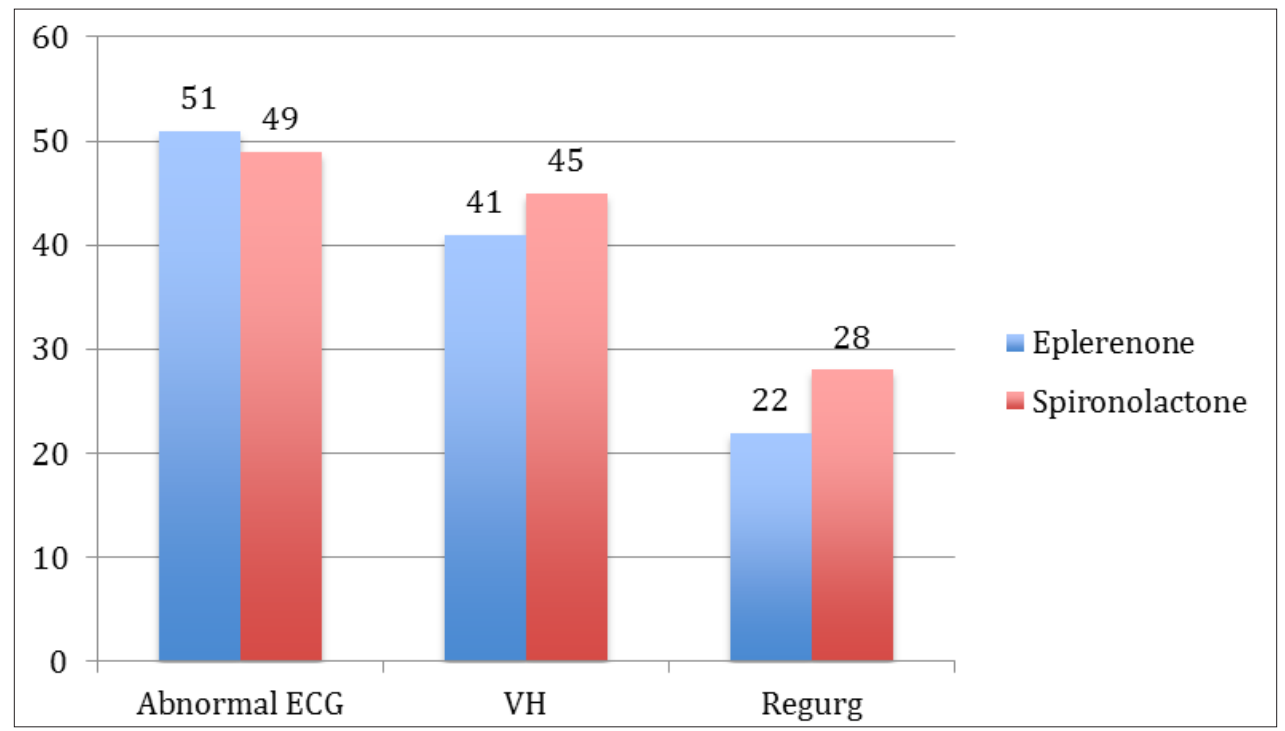

Figure 2.Eight-week Study Parameters among Sample Population

Table 6 shows the change in study parameters among treatment groups. Systolic blood pressure, diastolic blood pressure and CK levels showed a significant change over the study duration, with Eplerenone producing a better result than Spironolactone. Remaining other parameters showed a non-significant difference.

Table 6.Comparison of Change in Study Parameters among Treatment Groups

\begin{tabular}{|c|c|c|c|c|c|}
\hline & \multicolumn{2}{|c|}{ Eplerenone } & \multicolumn{2}{c|}{ Spironolactone } & \multirow{2}{*}{-value } \\
\cline { 2 - 5 } & Mean & SD & Mean & SD & \\
\hline Systolic BP & 132.8 & 9.43 & 133.1 & 9.3 & $0.031^{*}$ \\
\hline Diastolic BP & 92.43 & 4.35 & 92.73 & 4.22 & $0.048^{*}$ \\
\hline Blood Urea & 45.33 & 13.537 & 45.63 & 13.407 & 0.345 \\
\hline Serum Creatinine & 2.283 & 0.6002 & 2.583 & 0.4702 & 0.564 \\
\hline CK & 195.74 & 23.697 & 196.04 & 23.567 & $0.018^{*}$ \\
\hline Total Cholesterol & 246.08 & 39.242 & 248.18 & 38.542 & 0.364 \\
\hline HDL & 41.19 & 3.176 & 43.29 & 2.476 & 0.678 \\
\hline LDL & 138.69 & 30.437 & 140.79 & 29.737 & 0.543 \\
\hline Triglyceride & 199.38 & 28.423 & 201.48 & 27.723 & 0.788 \\
\hline SGOT & 35.918 & 6.7677 & 38.018 & 6.0677 & 0.867 \\
\hline SGPT & 57.74 & 11.605 & 59.84 & 10.905 & 0.962 \\
\hline LDH & 242.31 & 32.629 & 244.41 & 31.929 & 0.138 \\
\hline Alkaline Phosphatase & 126 & 20.426 & 128.1 & 19.726 & 0.978 \\
\hline
\end{tabular}

\section{Discussion}

Aldosterone, a hormone of the renin-angiotensinaldosterone system, has been linked to hypertension, cardiac hypertrophy, and cardiac and vascular fibrosis. Despite treatment with an angiotensin-converting enzyme inhibitor or angiotensin-Il receptor blocker, suppression of aldosterone is incomplete due to non-angiotensinII regulators of aldosterone production, such as serum potassium. Until recently, the only approved aldosterone antagonist for the treatment of hypertension and heart failure was Spironolactone. ${ }^{9}$ Eplerenone selectively binds to the mineralocorticoid receptor, thereby blocking the binding of aldosterone and thus inhibiting sodium reabsorption and other deleterious aldosterone-mediated mechanisms. ${ }^{10}$
Most published clinical trials evaluated the efficacy of Eplerenone for the treatment of hypertension. No clinical trials directly compared Eplerenone and Spironolactone for heart failure. ${ }^{10}$ With this background, the present observational study was attempted to study the efficacy of Eplerenone in treatment of heart failure and compare it with Spironolactone. Comparison of reduction in blood pressure with two treatments showed relative reduction to be significantly higher with Eplerenone than Spironolactone in the current study. Similar results have been obtained in a 12-week multicenter, double blind, randomized, placebo-controlled, parallel-arm, and intention-to-treat trial. ${ }^{11}$ EPHESUS Trial on heart failure also presented with similar findings. ${ }^{12}$ 
Clearly, patients with chronic severe HFREF (NYHA class III-IV) and patients with HFREF early post-MI should receive mineralocorticoid receptor antagonists (MRAs) in addition to standard therapy including an ACEI or ARB and a $\beta$-blocker if there is no contraindication to their use such as a serum potassium $>5.0 \mathrm{meq} / \mathrm{L}$ or an estimated glomerular filtration rate $(e G F R)<30 / \mathrm{mL} / \mathrm{min} / 1.73 \mathrm{~m}^{2} .{ }^{13}$ Other parameters like ECG findings improved significantly with treatment with Eplerenone in the current study. The pharmacologic effects of Eplerenone (a selective aldosterone antagonist) have not been studied as extensively as those of Spironolactone, particularly in humans.

In rats given exogenous angiotensin-II and dietary sodium chloride, Eplerenone reduced myocardial hypertrophy, expression of markers of coronary inflammation, and myocardial necrosis. ${ }^{14}$ The LV ejection fraction (LVEF) also improved significantly from $36 \%$ to $54 \%$ with Eplerenone at 8 weeks of treatment. The LVEF increased in patients enrolled in EPHESUS from $33 \%$ at baseline. ${ }^{12}$ The possible explanation could be revascularization and recovery from the acute event. These findings have important consideration for choosing MRA in treatment of chronic $\mathrm{HF}$ with a reduced left-ventricular ejection fraction (HFREF) and in patients with HFREF early postmyocardial infarction (post MI).

Current guidelines in general do not distinguish between the uses of these two agents. Spironolactone however has a known side effect profile including breast pain and gynecomastia in males and menstrual irregularities in premenstrual females due to its relative non-specificity for the mineralocorticoid receptor and its effects on androgen and progesterone receptors. Eplerenone, due to its relatively higher specificity for the mineralocorticoid receptor, is devoid of these side effects and therefore better tolerated, at least in males. ${ }^{13}$ Hyperkalemia appeared to be an issue when Spironolactone became routine practice for treatment of HF. After the RALES study, Spironolactone tended to be confined to the sickest New York Heart Association class-III and IV heart failure, with careful monitoring. ${ }^{15}$

The present research also showed greater potassium balance with Eplerenone. This is contrary to the findings of EPHESUS trial wherein serum concentrations of potassium and creatinine increased in both groups, and the increases were greater in patients who received Eplerenone. Serious hyperkalemia was more frequent in the Eplerenone -treated patients $(5.5 \%)$ than in those treated with placebo (3.9\%) $(P=0.002) .{ }^{12}$ Even a study by Pitt et al. in 2003 demonstrated hyperkalemia to be a major side effect with the drug. ${ }^{16}$ However, direct comparison with Spironolactone is warranted in future research for comparison.

No significant improvement was observed with respect to lipid profile of the patients on treatment with the two drugs in our research. This is corroborating with the fact that Eplerenone has major adverse effect in the form of hypertriglyceridemia as reported in studies by Craft et al. ${ }^{9}$ A study by Goodfriend et al. showed a negative correlation between aldosterone and cardio-protective high-density lipoprotein (HDL) levels, suggesting that these effects are, at least in part, mediated through increased MR activation. ${ }^{17}$ However, in summary, the available data remains controversial and there is still no conclusive evidence that establishes aldosterone and MR activation as critical factors in lipid or glucose homeostasis. Different MR blockers, e.g., Spironolactone versus Eplerenone, have different pharmacokinetic profiles.

Therefore, the effect of MR blockade on metabolic parameters may vary by the MR antagonist applied. For example, Eplerenone has been shown to improve insulin resistance in animal models of obesity. Similarly, in a mouse model of diet-induced diabetes mellitus MR blockade by Spironolactone induced improvements in glucose and lipid metabolism. ${ }^{18,19}$ The present study did not show any significant improvement in kidney function tests with two drugs; however, the decline was more seen with Spironolactone. Similar findings have been reported elsewhere also. ${ }^{20}$ Blockade of the MR has been demonstrated to have a general reno-protective effect in patients with diabetes mellitus and with chronic kidney disease. Evidence suggests Spironolactone to be more effective in stabilizing renal function tests in comparison to other MRAs.

\section{Limitations of the Study}

- The study was restricted to observational design, which has its own limitations. Compliance was an issue which, however, stringently followed, will probably encounter a trace of error, leading to bias in outcome.

- A research group of 100 individuals may not be diagnostically sufficient for concluding such evidences. A larger sample size with preferably a cohort-based periodical evaluation would be more helpful.

- Other parameters, particularly related to cardiovascular abnormalities, would also have been helpful in judging the status of the chronic comorbidity.

\section{Conclusion}

Comparison of reduction in blood pressure with two treatments showed relative reduction to be significantly higher with Eplerenone than Spironolactone in the current study. Other parameters like ECG findings improved significantly with treatment with Eplerenone in the current study. No significant improvement was observed with respect to lipid profile of the patients on treatment with the two drugs in our research. The present study did not show any significant improvement in kidney function tests 
with two drugs; however, the decline was more seen with Spironolactone.

\section{Conflict of Interest: None}

\section{References}

1. Congestive Heart Failure. [Internet]. Available on: http://www.mercksource.com/pp/us/cns/cns_hl_ dorlands_split.jsp ?pg=/ppdo cs/us/common/dorlands/ dorland/four/000047501.htm.

2. Hobbs R, Boyle A. Heart Failure. [Internet]. Available on: www.clevelandclinicmed.com/medicalpubs/ diseasemanagement/cardiology/heart-failure.

3. Definition of Heart Failure. [Internet]. Available on: http://www.mayoclinic.com/health/heartfailureIDS00061.

4. Asscia C. Facts about congestive heart failure. [Internet]. Available on: www.cardioassoc.com/patientygs/ conditions/congestive.asp.

5. Kleiman J, Krause S, Burns D et al. Effects of Eplerenone, Enalapril, and Eplerenone/Enalapril in patients with essential hypertension and left ventricular hypertrophy: the 4E-left ventricular hypertrophy study. Circulation 2003; 108: 1831-38.

6. Satoskar RS et al. Pharmacology and Pharmacotherapeutics. Revised 23 ${ }^{\text {rd }}$ edition. Chap 38: 567-68.

7. Ray S, Gupta R. Strategies for chronic heart failure management. Association of Physicians of India Chap 30: 164-65.

8. Shaikh Z, Pathak R. Revised Kuppuswamy and BG Prasad socio-economic scales for 2016. Int J Community Med Public Health 2017; 4: 997-99.

9. Craft J. Eplerenone (Inspra), a new aldosterone antagonist for the treatment of systemic hypertension and heart failure. Proceedings Baylor University Medical Center 2004; 17(2): 217-20.

10. Inspra, Eplerenone [product information] Pfizer Pharmaceuticals Jun 2003.

11. White WB, Carr AA, Krause S et al. Assessment of the novel selective aldosterone blocker Eplerenone using ambulatory and clinical blood pressure in patients with systemic hypertension. Am J Cardiol 2003; 92: 38-42.

12. Flack JM, Oparil S, Pratt JH et al. Efficacy and tolerability of Eplerenone and losartan in hypertensive black and white patients. J Am Coll Cardiol 2003; 41: 1148-55.

13. Pitt B, Zannad F. Eplerenone: Is it time to add this drug to current heart failure therapy? Therapeutic Advances in Chronic Disease 2012; 3(1): 5-9.

14. Marcy TD, Ripley TR. Aldosterone antagonists in the treatment of heart failure. Am J Health Syst Pharm 2006; 63(1): 49-58.

15. Pitt B, Zannad F, Remme WJ et al. The effect of Spironolactone on morbidity and mortality in patients with severe heart failure. N Engl J Med 1999; 341: 709-17.

16. Pitt B, Reichek N, Willenbrock R, et al. Effects of Eplerenone, Enalapril, and Eplerenone/Enalapril in patients with essential hypertension and left ventricular hypertrophy: the 4E-left ventricular hypertrophy study. Circulation 2003; 108: 1831-38.

17. Goodfriend TL, Egan B, Stepniakowski K et al. Relationships among plasma aldosterone, highdensity lipoprotein cholesterol, and insulin in humans. Hypertension 1995; 25: 30-36.

18. Guo C, Ricchiuti V, Lian BQ et al. Mineralocorticoid receptor blockade reverses obesity-related changes in expression of adiponectin, peroxisome proliferatoractivated receptor-gamma, and pro-inflammatory adipokines. Circulation 2008; 117: 2253-61.

19. Wada T, Kenmochi H, Miyashita Y et al. Spironolactone improves glucose and lipid metabolism by ameliorating hepatic steatosis and inflammation and suppressing enhanced gluconeogenesis induced by high-fat and high-fructose diet. Endocrinology 2010; 151: 2040-49.

20. Mephisto Study Group. Effectiveness of Eplerenone or Spironolactone treatment in preserving renal function in primary aldosteronism. European Journal of Endocrinology 2013; 168: 75-81.

Date of Submission: 2018-04-02 Date of Acceptance: 2018-04-20 